\title{
The Impact of a Structured Clinical Pathway on the Application of Management Standards in Patients with Diabetic Ketoacidosis and Its Acceptability by Medical Residents
}

\author{
Imad S. Hassan ${ }^{*}$, Azzam D. Al-Otaibi' ${ }^{2}$, Meteb M. Al-Bugami ${ }^{3}$, Salih Bin Salih', \\ Yousef Al Saleh ${ }^{4}$, Salman Abdulaziz ${ }^{5}$ \\ ${ }^{1}$ Department of Medicine \& King Saud bin Abdulaziz University for Health Sciences, Riyadh, KSA \\ ${ }^{2}$ Department of Medicine, King Abdulaziz Medical City, Riyadh, KSA \\ ${ }^{3}$ Nephrology, Dalhousie University, Nova Scotia, Canada \\ ${ }^{4}$ Division of Endocrinology \& King Saud bin Abdulaziz University for Health Sciences, Riyadh, KSA \\ ${ }^{5}$ Internal Medicine \& Critical Care, Rashid Hospital, Dubai, UAE \\ Email: ${ }^{*}$ imadsahassan@yahoo.co.uk
}

Received 17 August 2014; revised 13 September 2014; accepted 6 October 2014

Copyright (C) 2014 by authors and Scientific Research Publishing Inc.

This work is licensed under the Creative Commons Attribution International License (CC BY).

http://creativecommons.org/licenses/by/4.0/

c) (i) Open Access

\section{Abstract}

Diabetic Ketoacidosis (DKA) is a serious and potentially a fatal complication of diabetes mellitus. Tools to guarantee proper, evidence-based, guideline implementation are of paramount importance and an essential element for quality patient care. Clinical pathways represent one such tool that clearly promotes the implementation of guidelines and research evidence into clinical practice. The aims of this study were to measure quantitatively and qualitatively the impact of a specially structured Resident-friendly, DKA clinical pathway on the application of evidence-based management standards and its acceptability by the treating resident physicians. A retrospective chart review of patients who were admitted prior to and after the launching of the clinical pathway and a questionnaire assessment of resident's acceptance of the pathway format were undertaken. Eighty one episodes of DKA in a total of 58 patients fulfilled the criteria for inclusion in the study. Thirty seven admissions were on the pathway $(45.7 \%)$ and 44 were not $(54.3 \%)$. Documentation of severity indices of patients who were admitted under the pathway were significantly improved with a trend for a shorter hospital stay. The duration of intravenous insulin therapy, intensive care unit consultation and diabetes educator involvement in patient care were not different between the two groups. Residents found the pathway user-friendly, educationally very valua-

${ }^{*}$ Corresponding author.

How to cite this paper: Hassan, I.S., et al. (2014) The Impact of a Structured Clinical Pathway on the Application of Management Standards in Patients with Diabetic Ketoacidosis and Its Acceptability by Medical Residents. Journal of Diabetes Mellitus, 4, 264-272. http://dx.doi.org/10.4236/jdm.2014.44038 
ble, reduced their workload and had a positive effect on their DKA management skills.Conclusions: Use of specially structured, resident-friendly pathway led to significant improvement in documentation of DKA severity indices and empowered our residents with evidence-based knowledge and skills to deal with this serious diabetic complication.

\section{Keywords}

Diabetic Ketoacidosis, Clinical Pathway, Trainees, Quality

\section{Introduction}

Diabetic Ketoacidosis (DKA) is a metabolic emergency characterized by the triad of hyperglycemia, high-anion gap metabolic acidosis and ketonemia. Despite its multifactorial etiology, DKA is known to be a preventable acute complication of diabetes mellitus specifically through patient education. Failure to appreciate and appropriately manage and prevent re-occurrence of DKA can lead to grave consequences. DKA is one of the most fearsome complications of diabetes, with mortality rates of up to $5 \%$ in developed countries [1] [2] up to $30 \%$ in less privileged health systems [3] and more than $90 \%$ when complicated by cerebral edema [4]. Death is usually attributed to co-morbidities, yet iatrogenic hypokalemia and fluid overload are sometimes responsible [5]. Therefore, the meticulous application of accepted management standards in DKA is an overriding priority. For this particular reason, various international, evidence-based guideline developers have recommended the use of specific hospital protocols for the care of these patients [1] [2]. Emphasis is put on prompt diagnosis, assessment of severity, decisions on sites of care, close monitoring, specific advice on timing of intravenous fluid and insulin therapy switch, preparation for discharge and multi-disciplinary care team involvement [1] [2]. Despite of the development and wide availability of DKA guidelines, implementation of management standards set by the guidelines were unfulfilled [6]-[8] with occasionally significant variation in the protocols of care between different health establishments [9]. Clinical pathways, also known as Integrated Care Pathways, represent a tool that clearly promotes the implementation of guidelines and research evidence into clinical practice. This was highlighted in a recent Cochrane's systematic review of the subject [10]. Over the years, clinical pathways have become an integral part of quality improvement in all health care disciplines and services [11]-[17]. Clinical Pathways (CPs) are structured, multidisciplinary care plans that detail the essential steps in the care of patients with a specific clinical problem [18] [19]. They organize, sequence, and time the major interventions both medical and non-medical (by nursing and other health care providers) that the patient should receive [18] [19]. The aim of this study is to compare the quality of management delivered to DKA patients prior to the era of DKA pathway to that one delivered after the application of the pathway. Additionally, we gauged the acceptability of the pathway by resident medical staff using a simple anonymous questionnaire.

\section{Methods}

Development of the Pathway:

The format of a DKA Clinical Pathway was devised by the department's Clinical Practice Guidelines Committee with the following goals in mind: First, it should, as a rule, contain practical information that has an impact on treatment outcome, length of stay, cost of care, and readmission rates. Secondly, it should be user friendly, and does not increase physician's workload. Thirdly, it should be built into daily patients' care and assists in a real time disease and patient management decisions. Fourthly, it should contain clear indicators on when a patient is ready for discharge and when and which specialty is to follow this patient. To achieve all these goals, the committee involved all disciplines involved in DKA care (Endocrine, Internal Medicine, Pharmacy, Intensive Care, Residents, Diabetes Educators and Nursing) in its development and preprinted the format on the regular sheet that residents use for admitting patients. Important practical advice was highlighted in a sidebar that followed the pertinent sections of history, examination, investigation, treatment, discharge, and follow-up etc. An admission and a discharge order sheets were also included to assist in guideline implementation. A copy of the pathway is enclosed.

Post-implementation Chart Review: 
A retrospective charts review of adult patients (more than 12 years old) who were admitted to hospital with DKA as the first-listed diagnosis was undertaken. The study divided the DKA patients to two groups: those who were admitted under DKA pathway (Pathway group), and those who were not (Non-pathway group) and compared the quality of management delivered to both groups using carefully selected parameters including:

1) The documentation of severity indices (e.g. anion gap, corrected sodium level, plasma osmolality).

2) The duration of intravenous insulin therapy.

3) The duration of hospital stay.

4) The number of intensive care unit consultations.

5) The involvement of the diabetes educator in patient care.

The diagnosis of DKA was made based on ADA 2003 diagnostic criteria for DKA (with some minor modifications). These criteria were: plasma glucose $>15 \mathrm{mmol} / \mathrm{l}$. arterial $\mathrm{pH}$ less than 7.30 , serum bicarbonate less than $18 \mathrm{~m} \mathrm{Eq} / \mathrm{l}$, positive serum or urine ketones and anion gap of more than 10. Co-morbidities that were documented were entered from the patient charts. Infection was confirmed if cultures were positive or if a clinical diagnosis of infection was made by the treating team.

Pathway Acceptability by Resident Physicians:

A survey form was send to all resident staff affiliated to the department of medicine. This was undertaken by the guidelines committee to gauge pathway acceptability three months after its launching. The questionnaire addressed the ease of using the pathway, its impact on workload, its educational and practical value and any suggestions for its amendments.

Data Analysis

Data was entered on a Minitab spreadsheet for analysis. Simple descriptive statistical tests were used together with the 2-sample t-test and Chi square test as appropriate to test for significance and associations. A p-value of less than 0.05 is considered significant and the actual value quoted.

\section{Results}

102 patient charts with a diagnosis of DKA over a period of thirty months were reviewed. 81 episodes of DKA in a total of 58 patients fulfilled the criteria for inclusion in the study. 37 admissions were on the pathway (45.7\%) and 44 were not (54.3\%). Female gender admissions constituted 53.1\% (43 admissions). The two groups were found to be comparable at time of admission regarding mean age, metabolic severity indices (i.e. anion gap, glucose levels, $\mathrm{pH}$ and $\mathrm{HCO}_{3}$ levels), the presence of concurrent infections and the presence of comorbidities (Table 1). However, the documentation quality of severity indices was found to be significantly different between the two groups in favor of the pathway group. For example the anion gap (AG) was found to be documented in 32 charts out of 37 in the pathway group (86.49\%), compared to 25 out of $44(56.82 \%)$ in the non-pathway group with significant P-value of 0.004 (Table 2). Likewise the corrected sodium was found to be documented in 27 charts out of 37 (72.97\%) in the pathway group compared to 2 out 44 (4.55\%) in the nonpathway group with a P-value of 0.00001 (Table 2). Plasma osmolality was documented in 28 charts out of 37 (75.7\%) in the pathway group compared to 3 out of 44(6.82) in the non-pathway group with a similarly significant P-value of 0.000001 (Table 2). The duration of hospital stay was 3.84 days on average in pathway group compared to 3.98 days in non-pathway group $(\mathrm{P}$-value $=0.85$ ) (Table 2$)$. Similarly the duration of intravenous insulin therapy has a mean of 1.21 days among pathway group and 1.08 days among non-pathway groups $(\mathrm{P}$-value $=0.55)($ Table 2). The study didn't find any significant statistical difference between the two groups regarding ICU consultations and diabetes educator involvement in patient care (Table 2). There was no mortality case in either group.

The results of the acceptability survey are shown in Table 3. Thirty six residents received the survey, 28 (77.8\%) returned the filled one. It was clear that the selected format for guidelines implementation has gained wide acceptability by most participants in the survey. Comments of residents on the pathway are shown on Table 4.

\section{Discussion}

In its model of guaranteeing effective knowledge transfer, the Agency for Health Research and Quality emphasizes a three-stage process the last of which is organizational adoption and implementation [20]. Clinical path- 
Table 1. Patients characteristics at admission.

\begin{tabular}{cccc}
\hline & Pathway group (n=37) & Non pathway group (n= 44) & P-Value \\
\hline Age: mean (SD) & $23.5(10.6)$ & $24(12.8)$ & 0.85 \\
Concurrent infection: no. (\%) & $8(21.6 \%)$ & $8(18.2 \%)$ & 0.699 \\
Co-morbidities: no. (\%) & $16(43.2 \%)$ & $15(34.1 \%)$ & 0.399 \\
Glucose level on admission (mmol/L): mean (SD) & $32.4(11)$ & $31.6(10)$ & 0.75 \\
pH: mean (SD) & $7.168(0.104)$ & $7.199(0.087)$ & 0.16 \\
$\mathrm{HCO}_{3}$ (mmol/L): mean (SD) & $9.30(4.27)$ & $10.24(4.05)$ & 0.32 \\
Anion gap: mean (SD) & $27.81(6.83)$ & $25.67(5.07)$ & 0.32 \\
\hline
\end{tabular}

Table 2. Documentation and interventions in the two groups.

\begin{tabular}{|c|c|c|c|}
\hline & Pathway group $(\mathrm{n}=37)$ & Non pathway group $(n=44)$ & P-Value \\
\hline Anion gap documented & $32(86.5 \%)$ & $25(56.8 \%)$ & 0.004 \\
\hline Corrected sodium documented & $27(73 \%)$ & $2(4.5 \%)$ & 0.000001 \\
\hline Plasma osomolality documented & $28(75.7 \%)$ & $3(6.8 \%)$ & 0.000001 \\
\hline ICU consultation & $7(18.9 \%)$ & $8(18.2 \%)$ & 0.932 \\
\hline Diabetes educator involvement & $28(75.7 \%)$ & $35(79.5 \%)$ & 0.676 \\
\hline Length of stay & 3.8 day (3.09) & 3.98 day (3.62) & 0.85 \\
\hline Duration of intravenous insulin infusion & 1.2 day $(0.85)$ & 1.1 day (1.03) & 0.85 \\
\hline
\end{tabular}

Table 3. Results of acceptability survey.

\begin{tabular}{|c|c|c|}
\hline & & Residents \\
\hline Number Participating & & 28 \\
\hline $\begin{array}{l}\text { Presentation of the pathway form: i.e. pre-printed in the admission sheet with practical and important } \\
\text { clinical information highlighted in the side bar. }\end{array}$ & Helpful (\%) & 100 \\
\hline Content of the pathway form: Amount of Information. & Adequate (\%) & 100 \\
\hline Practical use of the form: Is the pathway easy to use? & Yes $(\%)$ & 100 \\
\hline Practical use of the form (physician friendly or not?): Does it decrease your workload? & Yes $(\%)$ & $76.2^{*}$ \\
\hline Educational and practical value to patient care: Format is educational and practically helpful. & Yes $(\%)$ & 96.4 \\
\hline Educational and practical value to patient care: Format assists in guidelines implementation. & Yes (\%) & 96.3 \\
\hline Educational and practical value to patient care: Has the pathway changed your medical practice? & Yes $(\%)$ & 88.5 \\
\hline Reasonably flexible to accommodate the majority of relevant cases? & Yes (\%) & 80.8 \\
\hline In general, do you think that the current pathway format should be continued? & Yes $(\%)$ & 96.4 \\
\hline
\end{tabular}

*21 residents ticked the relevant boxes.

ways may clearly help in realizing this stage. The use of integrated care pathways for DKA was associated with a variable yet generally a significant improvement in quality service provision for this serious complication. In a study by Waller et al. [21] the use of an integrated DKA care pathway was associated with a significant improvement in the proportion of patients commencing intravenous insulin within 60 minutes of admission and a similar significant reduction in the prescription of antibiotics and low molecular weight heparin. Similarly, Ilag et al. [22] has shown that subjects on a DKA pathway were significantly more likely to receive the recommend- 
Table 4. Participants comments.

1. Educational and Practical Value:

i. Consultant Comments:

- $\quad$ Pathway has an educational value for residents so their future practice will be established on solid basis.

- $\quad$ The pathway ensures no action is missed in patient's care. It is quite educational to junior staff.

ii. Residents Comments:

- $\quad$ Complete plan for the patient is present from admission till follow-up (R1).

- $\quad$ Reminder of important issues during a heavy on-call (R4).

- $\quad$ Very useful (R1).

- Improved our assessment skills.

Truly the pathways improved our practice (R2).

ed intravenous fluid volume, education on sick-day management, and get an endocrine specialty consultation. A mandatory protocol for DKA management was likewise associated with significant decreases in intensive care and hospital lengths of stay and time to anion gap closure and ketone clearance [23]. Not all pathways or protocol goals were accomplished. For example a reduction of length of stay was not realized in the study by Waller et al. [21] and a study by Devalia [24] showed poor adherence to the DKA protocol. In this study, the use of the pathway with regards DKA patient management was associated with a significant improvement in at least the documentation of important severity indices namely anion gap, corrected sodium level and plasma osmolality. These are important parameters that have a direct impact on management decisions such as when to switch to subcutaneous insulin therapy and when to discontinue intravenous fluids. A more appropriate timing (possibly earlier) for these actions would be expected. However, no such beneficial effect was realized most likely because switch timing was already at international, high-quality recommendations. This also applies to our postpathway findings with regards to the length of stay, diabetes educator involvement and intensive care consultations. It has long being recognized that the magnitude of improvement in quality is directly proportional to how low the quality of service was at the time of a new and successfully implemented quality intervention [25].

Knowledge about the care of diabetes and its complication is generally not at an acceptable level for physicians under training with only 50\% asserting that they are fully confident in managing hypoglycemia [26] and only $43 \%$ confirming their confidence in dealing with DKA [27]. In our study, the pathway was preprinted in the admission sheet and designed in a way that helped the acting physician handle DKA patients in the most appropriate manner, starting from pertinent history taking hints, important examination pointers through practical immediate management steps and formulation of a care plan till discharge. Evidence-based recommendations and advice were provided in a sidebar in the same sheet. For our medical residents, who were exposed to management care plans portrayed as clinical pathways for the first time in their career and in the forgoing format, this endeavor was extremely acceptable and rewarding. The aims of the pathway as an educational tool and as a helpful reminder of important actions in busy on-calls (paper-based as opposed to computer-based electronic reminders), its ability to assist our junior resident staff to gain competence and experience faster and to help in decreasing their work load were clearly realized. These beneficial effects have been documented with the use of integrated care pathways in several other healthcare settings [10] [18]. The head of division of endocrinology comment that the DKA pathway has markedly reduced consultations to the division was another evidence of the last statement (personal communication). For the department and institute, the pathway has become a good source for acquiring research data and for audit. Other reported interventions to improve guideline implementation in DKA incorporate multidisciplinary interventions including knowledge assessment, individualized education and formal didactic teaching [28].

The main limitations of this study are inherent to its nature being a retrospective study and the relatively small patient numbers. Measures which are now in place to improve the implementation of the pathway include regular training on pathway use, the election of pathway champions from resident physician staff, the inclusion of the pathway in the intranet and in the institutes computerized physician order entry interface and the employment of a case manager to allow for better monitoring and implementation. 


\section{Conclusion}

Beside the academic advantages, the clinical pathways are known to decrease practice variations and improve several essential patient-, practicing physician- and hospital-related outcomes including satisfaction with the service, reduction of work-load, and cutting hospital care costs. The study has shown that the implementation of DKA clinical pathway has improved the patient care particularly with regard to the documentation of severity indices. Additionally, the value of the pathway in empowering our residents with proper evidence-base advice and knowledge for better patient care is validated.

\section{Acknowledgements}

The authors wish to acknowledge the valuable input of the Clinical practice Guidelines committee members namely Dr. Mohamed Al Moamary, Dr. Yousef Saleh, Dr. Khalid Al Khathlan, Dr. Sadoon Al Sadoon, Dr. Hind Al Ghadeer, Dr. Maha Al Ammari, Ms. Rehana Alothman, Dr. Muhammad Al Daker, Dr. Afaf Al Shammary, Dr. Sami Bashi, Dr. Mohammed Harakati and Dr. Salih Bin Salih. A special thanks goes to our residents for their invaluable contributions and comments. The authors confirm that there are no financial or other conflicts of interest that may bias their work or results.

\section{References}

[1] Kitabchi, A.E., Umpierrez, G.E., Miles, J.M. and Fisher, J.N. (2009) Hyperglycemic Crises in Adult Patients with Diabetes: A Consensus Statement from the American Diabetes Association. Diabetes Care, 32, 1335-1343. http://dx.doi.org/10.2337/dc09-9032

[2] Savage, M.W., Dhatariya, K.K., Kilvert, A., et al. (2011) Joint British Diabetes Societies Guideline for the Management of Diabetic Ketoacidosis. Diabetic Medicine, 28, 508-515. http://dx.doi.org/10.1111/j.1464-5491.2011.03246.x

[3] Mbugua, P.K., Otieno, C.F., Kayima, J.K., Amayo, A.A. and McLigeyo, S.O. (2005) Diabetic Ketoacidosis: Clinical Presentation and Precipitating Factors at Kenyatta National Hospital, Nairobi. East African Medical Journal, 82, S191S196.

[4] Laing, S.P., Swerdlow, A.J., Slater, S.D., et al. (1999) The British Diabetic Association Cohort Study, II: Causespecific Mortality in Patients with Insulin-Treated Diabetes Mellitus. Diabetic Medicine, 16, 466-471. http://dx.doi.org/10.1046/j.1464-5491.1999.00076.x

[5] Hamblin, P.S., Topliss, D.J., Chosich, N., Lording, D.W. and Stockigt, J.R. (1989) Deaths Associated with Diabetic Ketoacidosis and Hyperosmolar Coma, 1973-1988. Medical Journal of Australia, 151, 439-444.

[6] Singh, R.K., Perros, P. and Frier, B.M. (1997) Hospital Management of Diabetic Ketoacidosis: Are Clinical Guidelines Implemented Effectively? Diabetic Medicine, 14, 482-486. http://dx.doi.org/10.1002/(SICI)1096-9136(199706)14:6<482::AID-DIA371>3.0.CO;2-A

[7] Ferreri, R. (2008) Treatment Practices of Diabetic Ketoacidosis at a Large Teaching Hospital. Journal of Nursing Care Quality, 23, 147-154. http://dx.doi.org/10.1097/01.NCQ.0000313764.64149.6d

[8] Solá, E., Garzón, S., García-Torres, S., Cubells, P., Morillas, C. and Hernández-Mijares, A. (2006) Management of Diabetic Ketoacidosis in a Teaching Hospital. Acta Diabetologica, 43, 127-130. http://dx.doi.org/10.1007/s00592-006-0227-1

[9] Henriksen, O.M., Prahl, J.B., Røder, M.E. and Svendsen, O.L. (2007) Treatment of Diabetic Ketoacidosis in Adults in Denmark: A National Survey. Diabetes Research and Clinical Practice, 77, 113-119. http://dx.doi.org/10.1016/j.diabres.2006.10.013

[10] Rotter, T., Kinsman, L., James, E., et al. (2010) Clinical Pathways: Effects on Professional Practice, Patient Outcomes, Length of Stay and Hospital Costs. Cochrane Database of Systematic Reviews, 3, Article ID: CD006632.

[11] Corbelli, J.C., Janicke, D.M., Cziraky, M.J., Hoy, T.A. and Corbelli, J.A. (2009) Acute Coronary Syndrome Emergency Treatment Strategies: Improved Treatment and Reduced Mortality in Patients with Acute Coronary Syndrome Using Guideline-Based Critical Care Pathways. American Heart Journal, 157, 61-68. http://dx.doi.org/10.1016/j.ahj.2008.08.022

[12] ZuWallack, R.L. (2002) Critical Pathways for the COPD Exacerbation. European Respiratory Review, 12, $452-455$.

[13] Ganeshram, K. (2005) Integrated Care Pathways for Acute Stroke. Age Ageing, 34, 91. http://dx.doi.org/10.1093/ageing/afh225

[14] Napolitano, L.M. (2005) Standardization of Perioperative Management: Clinical Pathways. Surgical Clinics of North America, 85, 1321-1327. http://dx.doi.org/10.1016/j.suc.2005.10.011 
[15] Collins, C.G. and Leahy, A.L. (2008) Integrated Care Pathways in Surgery. Surgeon, 6, 69-70. http://dx.doi.org/10.1016/S1479-666X(08)80066-2

[16] Olsson, L.E., Karlsson, J. and Ekman, I. (2007) Effects of Nursing Interventions within an Integrated Care Pathway for Patients with Hip Fracture. Journal of Advanced Nursing, 58, 116-125. http://dx.doi.org/10.1111/j.1365-2648.2007.04209.x

[17] Luhrs, C.A. and Penrod, J.D. (2007) End-of-Life Care Pathways. Current Opinion in Supportive and Palliative Care, 1, 198-201. http://dx.doi.org/10.1097/SPC.0b013e3282f1aae6

[18] Campbell, H., Hotchkiss, R., Bradshaw, N. and Porteous, M. (1998) Integrated Care Pathways. British Medical Journal, 316, 133-137. http://dx.doi.org/10.1136/bmj.316.7125.133

[19] Kinsman, L., Rotter, T., James, E., Snow, P. and Willis, J. (2010) What Is a Clinical Pathway? Development of a Definition to Inform the Debate. BMC Medicine, 8, 31. http://dx.doi.org/10.1186/1741-7015-8-31

[20] Nieva, V.F., Murphy, R., Ridley, N., et al. (2005) From Science to Service: A Framework for the Transfer of Patient Safety Research into Practice. In: Advances in Patient Safety: From Research to Implementation, Agency for Healthcare Research and Quality, Rockville.

[21] Waller, S.L., Delaney, S. and Strachan, M.W. (2007) Does an Integrated Care Pathway Enhance the Management of Diabetic Ketoacidosis? Diabetic Medicine, 24, 359-363. http://dx.doi.org/10.1111/j.1464-5491.2007.02102.x

[22] Ilag, L.L., Kronick, S., Ernst, R.D., Grondin, L., Alaniz, C., Liu, L. and Herman, W.H. (2003) Impact of a Critical Pathway on Inpatient Management of Diabetic Ketoacidosis. Diabetes Research and Clinical Practice, 62, $23-32$. http://dx.doi.org/10.1016/S0168-8227(03)00143-8

[23] Bull, S.V., Douglas, I.S., Foster, M. and Albert, R.K. (2007) Mandatory Protocol for Treating Adult Patients with Diabetic Ketoacidosis Decreases Intensive Care Unit and Hospital Lengths of Stay: Results of a Nonrandomized Trial. Critical Care Medicine, 35, 41-46. http://dx.doi.org/10.1097/01.CCM.0000249825.18677.D2

[24] Devalia, B. (2010) Adherence to Protocol during the Acute Management of Diabetic Ketoacidosis: Would Specialist Involvement Lead to Better Outcomes? International Journal of Clinical Practice, 64, 1580-1582. http://dx.doi.org/10.1111/j.1742-1241.2010.02348.x

[25] Titler, M.G. (2008) Chapter 7: The Evidence for Evidence-Based Practice Implementation. In: Hughes, R.G., Ed., Patient Safety and Quality: An Evidence-Based Handbook for Nurses, Agency for Healthcare Research and Quality (US), Rockville.

[26] George, J.T., Warriner, D.A., Anthony, J., Rozario, K.S., Xavier, S., Jude, E.B. and McKay, G.A. (2008) Training Tomorrow's Doctors in Diabetes: Self-Reported Confidence Levels, Practice and Perceived Training Needs of PostGraduate Trainee Doctors in the UK. A Multi-Centre Survey. BMC Medical Education, 8, 22. http://dx.doi.org/10.1186/1472-6920-8-22

[27] George, J.T., Warriner, D., McGrane, D.J., et al. (2011) Lack of Confidence among Trainee Doctors in the Management of Diabetes: The Trainees Own Perception of Delivery of Care (TOPDOC) Diabetes Study. QJM, 104, 761-766. http://dx.doi.org/10.1093/qjmed/hcr046

[28] Volkova, N.B., Fletcher, C.C., Tevendale, R.W., Munyaradzi, S.M., Elliot, S. and Peterson, M.W. (2008) Impact of a Multidisciplinary Approach to Guideline Implementation in Diabetic Ketoacidosis. American Journal of Medical Quality, 23, 47-55. http://dx.doi.org/10.1177/1062860607311015 


\section{Supplement}

A copy of Diabetic Ketoacidosis Clinical Pathway

\section{DIABETIC KETOACIDOSIS CLINICAL PATHWAY}

"This clinical path is a general guideline to assist in the management of patients. This guideline is not designed to replace clinical judgment or individual patient needs."

$\begin{array}{lll}\text { Admission Date: } & \text { Time: } & \text { Admitted From: ECC } \square \text { ACC } \square \text { Transfer } \square \\ \text { No Code } \square \text { Date: } & \text { Age: } & \text { M } \square \text { F } \square \text { Consultant: }\end{array}$

ESSENTIAL INFORMATION FOR DKA ADMISSIONS

$\checkmark \quad$ Diagnosis of DKA confirmed: $\quad$ Yes $\square$ No $\square$

Criteria for Diagnosis:

Blood Glucose $>13.8 \mathrm{mmol} / \mathrm{dl}, \mathrm{pH}<7.3$, Serum Bicarbonate $<18 \mathrm{mmol} / \mathrm{L}$, Urine/Serum Ketones +ve, Anion Gap $>10$

\footnotetext{
$\square \quad$ Anion Gap: $\underline{\mathrm{mNa}^{+}}-\left(\mathrm{HCO}_{3}+\mathrm{Cl}^{-}\right)=$

$\square$ Corrected Sodium: (Se Glu-5.0)/5.0 × $1.5+$ Se Na $=$

Calculated Osmolality: $(2 \times \underline{\mathrm{mNa}}+$ Glucose + Urea $)=$
}

\section{$\checkmark \quad$ Assessment of Severity: Indications for Hospital Admission and Site of Care (History, Physical Examination, Investigations)}

PR....... RR..........BP Sup:...... Erect:... T Temp........Pulse Oximetry.......... On RA

\section{RISK STRATIFICATION OF PATIENTS WITH DKA}

\begin{tabular}{|c|c|c|c|c|}
\hline Severity & Plasma Glucose (mmol/dl) & Arterial pH & Serum Bicarbonate $(\mathrm{mEq} / \mathrm{L})$ & Anion Gap (mEq/L) \\
\hline Mild $\square$ & $>13.8$ & $7.25-7.3$ & $15-18$ & $>10$ \\
\hline Moderate $\square$ & $>13.8$ & $7-7.24$ & $10-14$ & $>12$ \\
\hline Severe $\square$ & $>13.8$ & $<7$ & $<10$ & $>12$ \\
\hline
\end{tabular}

\section{Presence of any of the Following Features makes ICU Admission Obligatory}

ICU admission for CVP monitoring is recommended in patients with history of significant cardiac or renal impairment/or with persistent oliguria or anuria*.

Altered level of consciousness

Respiratory rate $>35 / \mathrm{min}$

Refractory hypotension

Need for mechanical ventilation

Plasma osmolality is $>320$

$\mathrm{pH}$ at 7.0 or below

Hypokalemia at the time of presentation 


\section{Identify the precipitant for the acute attack:}

New diagnosis of DM $\square \quad$ Acute infection $\square \quad$ Lack of medications
$\square \quad$ Poor compliance to treatment $\square \quad$ Acute stress (CVA, ACS etc.) $\square \quad$ Pregnancy
$\square \quad$ Exposure to Meds $\square \quad$ Other (specify)

DKA Immediate Management: Treatment of DKA includes administration of intravenous fluid to correct dehydration and hyperosmolarity, administration of insulin to reverse hyperglycemia and ketoacidosis, correction of electrolyte abnormalities, identification of precipitants, and frequent patient monitoring. Complications of treatment include hypoglycemia; hypokalemia; and rarely, cerebral edema.

IV Fluid and Insulin Infusion Switch: Criteria for switch include: patient is fully conscious, able to take orally, first sc

Promote self-management plan/Home Blood Glucose Monitoring/ home ketostix for those with frequent DKAs.

insulin dose given one to two hours earlier, $\mathrm{HCO}_{3}^{-}>18 \mathrm{mmol} / \mathrm{l}$, anion gap $<14$ and $\mathrm{pH}>7$.3. Start with patient's usual insulin regimen, adjusted if need be. New patient: Sc insulin at a total daily dose of 0.7 unit/kg divided as follow: $-2 / 3$ for am dose (1/3 $\mathrm{R} \& 2 / 3 \mathrm{NPH}),-1 / 3$ for pm dose (1/2 R \& 1/2 NPH)

Discharge: If the patient has met the above criteria, is established on a reasonably adequate insulin regimen, precipitant of DKA resolved or under control and is seen by the diabetes educator.

Follow-up: in endocrine clinic in 2 - 4 weeks. 
Scientific Research Publishing (SCIRP) is one of the largest Open Access journal publishers. It is currently publishing more than 200 open access, online, peer-reviewed journals covering a wide range of academic disciplines. SCIRP serves the worldwide academic communities and contributes to the progress and application of science with its publication.

Other selected journals from SCIRP are listed as below. Submit your manuscript to us via either submit@scirp.org or Online Submission Portal.
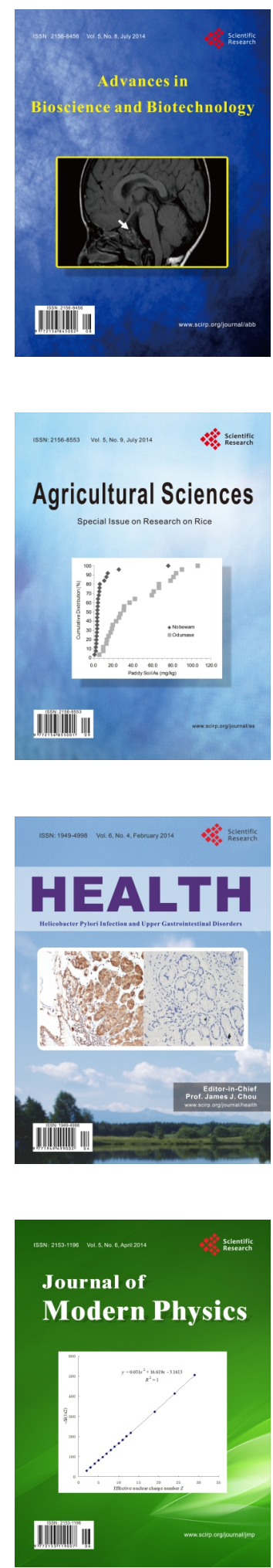
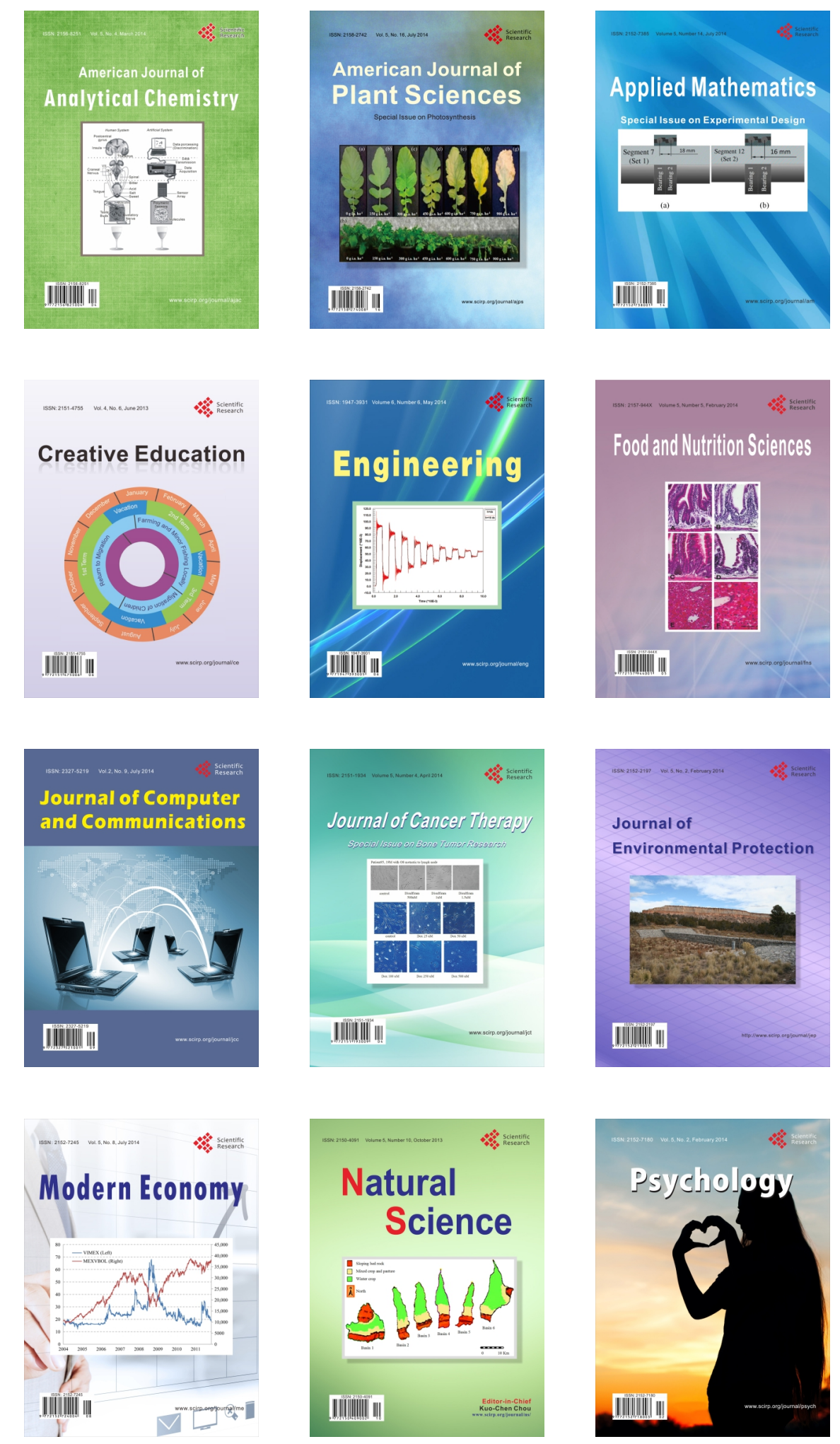